\title{
On the vorticity behind 3-D detached bow shock wave
}

\author{
V. N. Golubkin ${ }^{1,2^{*}}$ and G. B. Sizykh ${ }^{2}$
}

\author{
* Correspondence: valeriy.golubkin@ \\ tsagi.ru \\ ${ }^{1}$ Central Aerohydrodynamic \\ Institute named after professor N.E., \\ Zhukovsky, Russia \\ ${ }^{2}$ Moscow Institute of Physics and \\ Technology (State University), \\ Dolgoprudny, Russia
}

\begin{abstract}
For the general case of a spatial isoenergetic flow of ideal gas, Helmholtz's theorems are generalized and the speed with which vortex tubes move is found, keeping the intensity. It is shown that along the streamline without stagnation point, vorticity either is equal to zero everywhere, or it is non zero at all. The pattern of vortex lines behind the three-dimensional detached bow shock wave is specified.
\end{abstract}

Keywords: Vortex Helmholtz's theorems, Crocco theorem, Isoenergetic flows, Vorticity, Detached bow shock wave

\section{Introduction}

At flow with a uniform supersonic stream, the detached bow is formed in front of a body with the blunted nose part or with a high angle in a forward angular point, exceeding the marginal value up to which the attached shock is possible. For the specified reasons, the surface of such a shock is curved and generates vortex gas flow behind them. Properties of vorticity on the surface of curved shock waves near bluff bodies are rather well studied (see Refs. $[1,2]$ ). For the flow behind the shock, the main known result consists that in a plane case the ratio of vorticity to pressure $I_{1}=\Omega / p$ is constant along streamlines, and in axisymmetric case the analogous invariant is $I_{2}=\Omega /(\mathrm{pr}), \mathrm{r}$ being the distance from axis of symmetry [3]. In Ref. [4], it was shown that for any fluid or gas flow, along with the real flow velocity $\mathbf{u}$, there is a speed $\mathbf{U}$ of some imagined environment whose particles transfer vortex tubes (that is vector tubes of the vorticity field) with maintaining their intensity. Classical theorems of Helmholtz [5] show that for barotropic ideal fluid fictitious speed $\mathbf{U}$ coincides with the real flow velocity $\mathbf{u}$.

In Ref. [4], the method of determination of the speed $\mathbf{U}$ for any flow is also suggested. But this approach is not local, because calculation of the velocity field $\mathbf{U}$ requires integration of hydrodynamic functions along vortex lines. So far local methods to calculate $\mathbf{U}$ were known only for two types of flows. The first one - already mentioned barotropic ideal fluid flows, where $\mathbf{U}=\mathbf{u}$. The second type - the plane-parallel and axisymmetric nontwisted flows of viscous incompressible fluid, for which according to Refs. [6, 7]

$$
\mathbf{U}=\mathbf{u}-v[\mathbf{\Omega} \times[\nabla \times \mathbf{\Omega}]] / \mathbf{\Omega}^{2},
$$

where $v$ is kinematic viscosity coefficient. The new class of flows for which it is possible to offer a local method to calculate $\mathbf{U}$ is found in this paper.

(c) The Author(s). 2019 Open Access This article is distributed under the terms of the Creative Commons Attribution 4.0 International License (http://creativecommons.org/licenses/by/4.0/), which permits unrestricted use, distribution, and reproduction in any medium, provided you give appropriate credit to the original author(s) and the source, provide a link to the Creative Commons license, and indicate if changes were made. 
Helmholtz's theorems aren't valid for non barotropic flows. In particular, such flows may be isoenergetic that is supposed further. Flows behind detached shock waves in front of blunted bodies in a uniform supersonic stream belong to this class. Since the surface of detached shock is curved, the entropy of gas crossing it gains various entropy values on different streamlines, making a flow non barotropic and, according to Crocco theorem [8], - vortex. However, it has turned out that for such flows, analogs of Helmholtz theorems are valid. Expression of speed of vortex tubes motion with maintaining their intensity is found in this paper, and it is shown that all vortex lines behind the shock are closed and once cover the streamline, beginning in the shock point where the tangent plane is orthogonal to the free stream direction.

\section{Equations of gas motion}

The relationship between gas pressure $p$ and density $\rho$ has the form $p=\sigma \rho^{k}$, where $-k$ is the ratio of specific heats, $\sigma$ is entropy function which is constant along streamlines and for the flows considered, has different values on various streamlines. At the same time, such flow is isoenergetic, that is, the total enthalpy (energy) $k(k-1)^{-1} p \rho^{-1}+u^{2} / 2$ is constant everywhere. Crocco theorem [8] being in our case

$$
\mathbf{\Omega} \times \mathbf{u}=(k-1)^{-1} p \rho^{-1} \nabla \ln \sigma,
$$

is supplemented with the continuity equation

$$
\operatorname{div}(\rho \mathbf{u})=0 .
$$

Gas dynamic functions $\mathbf{u}, \rho$ and $p$, are supposed to be twice continuously differentiable on spatial coordinates.

\section{Vortex alternative}

Equation (1) may be written as

$$
\left(p^{-1} \mathbf{\Omega}\right) \times(\rho \mathbf{u})=(k-1)^{-1} \nabla \ln \sigma .
$$

Applying the rotor operation to both parts of this equation, taking into account eq. (2) and equality div $\boldsymbol{\Omega}=0$, valid since vorticity $\boldsymbol{\Omega}=\operatorname{rot} \mathbf{u}$, gives

$$
\rho(\mathbf{u} \cdot \nabla)\left(p^{-1} \mathbf{\Omega}\right)-\left(\left(p^{-1} \boldsymbol{\Omega}\right) \cdot \nabla\right)(\rho \mathbf{u})-\rho \mathbf{u}\left(\left(p^{-1} \mathbf{\Omega}\right) \cdot \nabla \ln p\right)=0 .
$$

The flow velocity is represented in the form $\mathbf{u}=u \mathbf{e},|\mathbf{e}|=1$. Under the condition $u \neq 0$, the last equation is equivalent to the following one

$$
(\mathbf{e} \cdot \nabla)\left(p^{-1} \boldsymbol{\Omega}\right)-\left(\left(p^{-1} \boldsymbol{\Omega}\right) \cdot \nabla\right) \mathbf{e}-\mathbf{e}\left(\left(p^{-1} \boldsymbol{\Omega}\right) \cdot \nabla \ln (p \rho u)\right)=0 .
$$

Let's consider an arbitrary streamline with the vorticity $\boldsymbol{\Omega}=\boldsymbol{\Omega}(l)$, where $l$ is variable arc length along this streamline. Then we may write

$$
(\mathbf{e} \cdot \nabla)\left(p^{-1} \mathbf{\Omega}\right)=\frac{d}{d l}\left(p^{-1} \boldsymbol{\Omega}\right) .
$$

Denote $e_{x}, e_{y}, e_{z}$ and $\Omega_{x}, \Omega_{y}, \Omega_{z}$ unit vector $\mathbf{e}$ and vorticity vector $\Omega$ components in some Cartesian coordinate system $O x y z, F=\ln (p \rho u)$. Using these notations and equality (4) it is possible to write vector eq. (3) along the streamline considered in the matrix form: 


$$
\frac{d}{d l}\left(\begin{array}{c}
\frac{\Omega_{x}}{p} \\
\Omega_{y} \\
\frac{p}{\Omega_{z}} \\
\frac{p}{p}
\end{array}\right)=\left(\begin{array}{ccc}
\frac{\partial}{\partial x} e_{x}+e_{x} \frac{\partial}{\partial x} F & \frac{\partial}{\partial y} e_{x}+e_{x} \frac{\partial}{\partial y} F & \frac{\partial}{\partial z} e_{x}+e_{x} \frac{\partial}{\partial z} F \\
\frac{\partial}{\partial x} e_{y}+e_{y} \frac{\partial}{\partial x} F & \frac{\partial}{\partial y} e_{y}+e_{y} \frac{\partial}{\partial y} F & \frac{\partial}{\partial z} e_{y}+e_{y} \frac{\partial}{\partial z} F \\
\frac{\partial}{\partial x} e_{z}+e_{z} \frac{\partial}{\partial x} F & \frac{\partial}{\partial y} e_{z}+e_{z} \frac{\partial}{\partial y} F & \frac{\partial}{\partial z} e_{z}+e_{z} \frac{\partial}{\partial z} F
\end{array}\right)\left(\begin{array}{c}
\frac{\Omega_{x}}{p} \\
\frac{\Omega_{y}}{p} \\
\frac{\Omega_{z}}{p}
\end{array}\right)
$$

The matrix elements depend on both gasdynamic functions themselves and on their first derivatives, and are continuous (and limited) functions along arbitrary streamline, where velocity value $u \geq u_{0}>0$ with any arbitrary positive amount $u_{0}>0$. If we consider the matrix elements to be given functions of the variable $l$, matrix eq. (5) represents the system of scalar ordinary differential equations for the vector $p^{-1} \Omega$ components. This system is linear, its coefficients being continuous and limited. Hence, from the theorem of existence and uniqueness for systems of ordinary differential equations, it follows that along all the streamline considered either $\left|p^{-1} \boldsymbol{\Omega}\right| \equiv 0$, or $\left|p^{-1} \boldsymbol{\Omega}\right| \neq 0$. Since the above reasoning is valid for an arbitrary value $u_{0}>0$, we come to the following conclusion named vortex alternative:

If velocity value isn't zero along some streamline of steady isoenergetic flow, then the vorticity value $|\Omega|$ either identically equals zero or $|\Omega| \neq 0$ along all the line.

\section{Speed of vortex tubes transfer}

Using the expression for entropy function we rewrite eq. (1) as follows $\boldsymbol{\Omega} \times($ $\left.\left(\rho / \rho_{0}\right)^{1-k} \mathbf{u}\right)=(k-1)^{-1} \rho_{0}^{k-1} \nabla \sigma$,

where $\rho_{0}$ is some reference gas density (for instance, in free supersonic stream). Applying rotor operation of the both sides, bearing in mind vorticity field solenoidal property $(\operatorname{div} \boldsymbol{\Omega}=0)$, we find that the function

$$
\mathbf{U}=\left(\rho / \rho_{0}\right)^{1-k} \mathbf{u}
$$

satisfies the following condition $\nabla \times[\boldsymbol{\Omega} \times \mathbf{U}]=0$.

According to Zorawski criterion (Ref. [9]), we obtain the following generalization of classical Helmholtz theorems:

In nonbarotropic isoenergetic gas flow vortex lines and vortex tubes move at the speed $\mathbf{U}$, and the intensity of vortex tubes is preserved.

Note that vortex lines are immovable in the steady flow considered, and the function $\mathbf{U}$ should be considered as the speed of imaginary environment particles. Its particles which constitute vortex tube at some time moment and move at the speed $\mathbf{U}$ will constitute the vortex tube of the same intensity at every subsequent moment. Thus choosing different $\rho_{0}$ values we shall "observe" motion of fictitious particles at different speeds, while the steady vorticity field pattern naturally will be the same.

\section{Vortex lines behind detached shock wave}

We consider a smooth convex body in a uniform supersonic stream with the formation of detached shock wave. We arrange the rectangular Cartesian system of coordinates Axyz having the origin in some point on the shock so that its $z$ axis coincides with a normal to the shock surface. Considering that the shock also has the smooth form and a condition of continuity of a tangent velocity component, it is simple to be convinced 
that the normal vorticity component has the same property of continuity. As the vorticity in uniform free supersonic stream is equal to zero, the normal vorticity component on both sides of the shock is also equal to zero, that is, vortex lines lie on the shock.

It follows from the eq. (1) that vortex lines lie on isentropic surfaces $\sigma=$ const. Entropy function on the shock surface has the same value on the line along which the normal to jump makes the same angle with a vector of free stream speed. Such lines lying on the shock of a smooth convex shape, are closed and encompass the only point in which the vector of a normal is parallel to the free stream speed (see Fig. 1).

Further for brevity this point is called leading point. It is obvious that the stream surfaces beginning on the above mentioned closed lines, represent isentropic surfaces $\sigma=$ const, on which, as also noted above, vortex lines lie. The result obtained in the previous section allows to specify vision of vortex lines pattern. Really, particles of the imagined fluid which make the closed vortex lines lying on the shock in some time moment move downstream with a speed $\mathbf{U}=\left(\rho / \rho_{0}\right)^{1-k} \mathbf{u}$ and continue to make vortex lines (see Fig. 2).

Owing to the continuity of the speed $\mathbf{U}$, we come to the following conclusion:

Behind the curved shock wave all vortex lines are closed and encompass the streamline beginning at the shock leading point.

This property is valid for both subsonic and supersonic flow domains behind the shock.

One more important property follows from a vortex alternative (see Section 3). Since the leading point vorticity is zero, it is equal to zero on the whole streamline leaving the leading shock point.

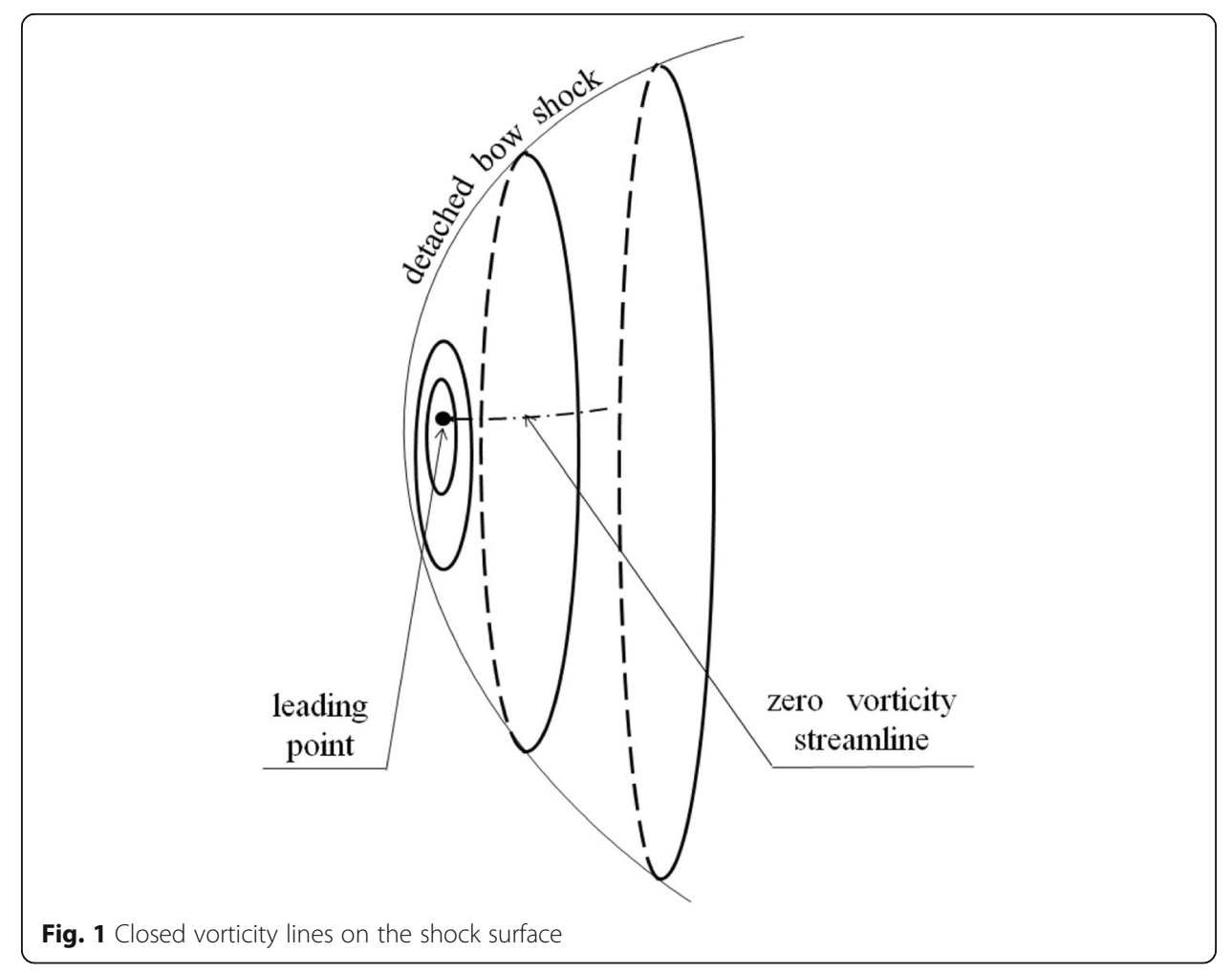




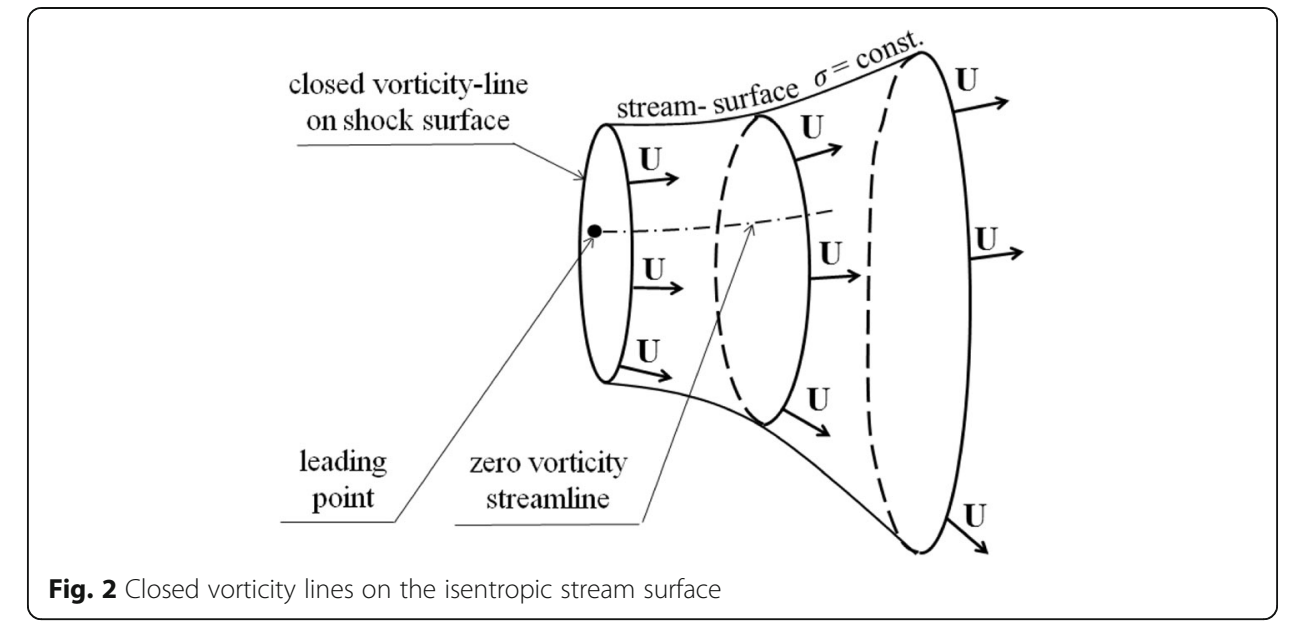

\section{Conclusion}

Based on the analysis of the full 3D Euler equations of steady non barotropic isoenergetic flows of ideal gas, generalization of Helmholtz theorems is obtained and expression for the speed of vortex tubes motion with maintaining their intensity is founded. It is shown that on each streamline without stagnation points, vorticity either is identically equal to zero, or doesn't equal to zero at all (a vortex alternative). It is also shown that in the general spatial case, the field of vorticity behind the curved shock wave having the leading point is characterized by the following properties:

1. Vortex lines are closed and only once cover the streamline beginning in the shock leading point.

2. Vorticity is zero along the streamline leaving the shock leading point.

Acknowledgements

The authors are grateful to the specialists of MIPT and TSAGl for discussion of results and general attention, in particular A.V. Wolkov.

Authors' contributions

Every author contributes equal part to the work about 50\%. All external informational sources are carefully referenced. Both authors read and approved the final manuscript.

\section{Authors' information}

Valeriy N. Golubkin, Professor, Doctor of Technical Sciences, TsAGI/MIPT, author of over 120 scientific publications. Research interests include high-speed aerodynamics, gas- and hydrodynamics. E-mail: vgolubkin@ou-link.ru. Grigory B. Sizykh, Associate Professor, Candidate of Physical-Mathematical Sciences, MIPT, author of over 40 scientific publications. Research interests include mathematical methods in gas- and hydrodynamics. E-mail: 010203@mail.ru.

\section{Funding}

Not applicable.

Availability of data and materials

All data obtained or analyzed in the course of this study are included in this published paper.

Competing interests

There are no financial or non-financial competing interests in the publication of the paper.

Received: 19 March 2019 Accepted: 6 June 2019

Published online: 08 July 2019

\section{References}

1. Truesdell C (1952) On curved shocks in steady plane flow of an ideal fluid. J Aeronaut Sci 19:826-828

2. Hayes WD (1957) The vorticity jump across a gasdynamic discontinuities. J Fluid Mech 2:595-600

3. Crocco L (1937) Eine neue Stromfunktion für die Erforschung der Bewegung der Gase mit Rotation. ZAMM 17(1):1-7 
4. Markov W, Sizykh GB (2015) Vorticity evolution in liquids and gases. Fluid Dyn 50(2):186-192

Kochin NE, Kibel' IA, Roze NV (1964) Theoretical hydromechanics. Interscience Publishers, New York

Golubkin VN, Sizykh GB (1987) Some general properties of plane-parallel viscous flows. Fluid Dyn 22(3):479-481

7. Brutyan MA, Golubkin VN, Krapivsky PL (1988) On Bernoulli equation for axisymmetric flow of viscous fluid. TSAGI Sci Notes 19(2):98-100 (in Russian)

8. Von Mises R (1958) Mathematical theory of compressible fluid flow. Academic Press, New York

9. Truesdell C (1954) The kinematics of vorticity. Indiana University Press, Bloomington

\section{Publisher's Note}

Springer Nature remains neutral with regard to jurisdictional claims in published maps and institutional affiliations.

Submit your manuscript to a SpringerOpen ${ }^{0}$ journal and benefit from:

- Convenient online submission

- Rigorous peer review

- Open access: articles freely available online

High visibility within the field

Retaining the copyright to your article 